\title{
Parotid lipoblastoma in a child: Rare presentation as huge infratemporal mass with cervical extension
}

\author{
Natarajan Anantharajan, Nagamuttu Ravindranathan \\ Department of Maxillofacial and Facial Plastic and Reconstructive Surgery, RIPAS Hospital, Brunei BA1710 Darussalam
}

Address for correspondence: Mr. Natarajan Anantharajan, 88-X, Molayanoor (Post), Pappireddipatti (Taluk), Dharmapuri (Dist.), Tamil Nadu, India. E-mail: nsanandms@yahoo.com

\section{ABSTRACT}

Lipoblastomas arising within the parotid gland and extending into the infratemporal fossa are very rare. They are common in children $<3$ years of age. Access to the tumour requires careful planning and interpretation of imaging studies. Lipoblastomas tend to recur. Meticulous dissection of the tumour is needed to prevent recurrence. By adopting the appropriate approach, complete removal is possible with minimal morbidity.

\section{KEY WORDS}

Facial nerve; infratemporal access; lipoblastoma; magnetic resonance angiogram; parotid tumour

\section{CASE REPORT}

3-year-old child presented to the Department of Maxillofacial and Facial Plastic Surgery with a history of painless swelling of 3 months duration in the right preauricular region.

Within a period of 6 months, the swelling rapidly increased in size and extended superiorly up to the temporal fossa and inferiorly with a huge cervical mass and medially with a buccal and parapharyngeal extension [Figure 1a and b]. The child had no facial weakness and no respiratory distress. On physical examination, the mass appeared smooth and soft overall except for a small region over the cervical part that appeared cystic (pseudofluctuation). Magnetic resonance imaging (MRI) showed a hyperintense mass on T1-weighted sequence and T2-weighted sequence in the right infratemporal fossa extending along the ramus of the mandible and infiltrating the parotid gland [Figure $2 \mathrm{a}$ and $\mathrm{b}$ ]. Magnetic resonance angiogram was performed to determine the relationship of the tumour with the carotid vessels. It showed stretching of the right external carotid artery around the mass with no significant vascularity [Figure 3]. Computerized tomogram (CT) scan showed a lobulated hypodense mass in the right infratemporal region (HU values -50 - to -75 , indicative of fat) with solid component [Figures 4 and 5]. In the CT scan, the mass was found displacing the parapharyngeal fat plane medially. Fine needle aspiration cytology of the mass demonstrated primarily adipose tissue with no evidence of malignant cells. Surgical removal for definitive diagnosis and to prevent cosmetic deformity was carried out.

\section{Operative Procedure}

At the start of the procedure, temporary tracheostomy was performed to secure the airway. The operation was carried out via a pre-auricular incision with submandibular extension [Figure $6 \mathrm{a}$ and $\mathrm{b}$ ]. The submandibular gland with duct was removed, preserving the lingual and hypoglossal nerve as surgical access to infratemporal fossa was achieved from below. Lower lip split was carried 


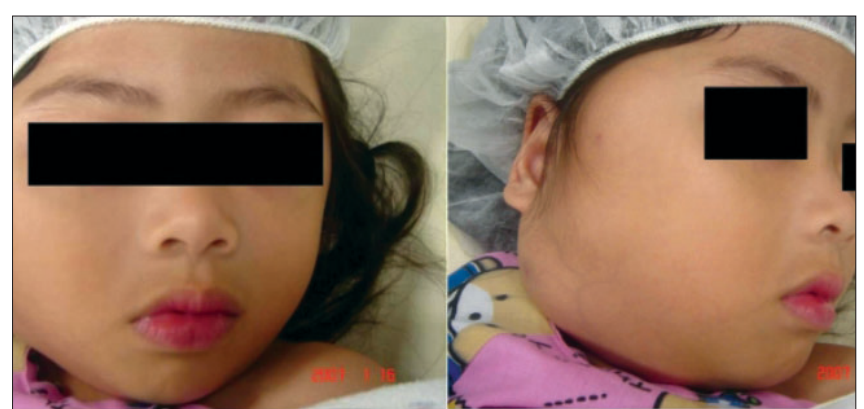

Figure 1: Clinical photograph showing the lesion

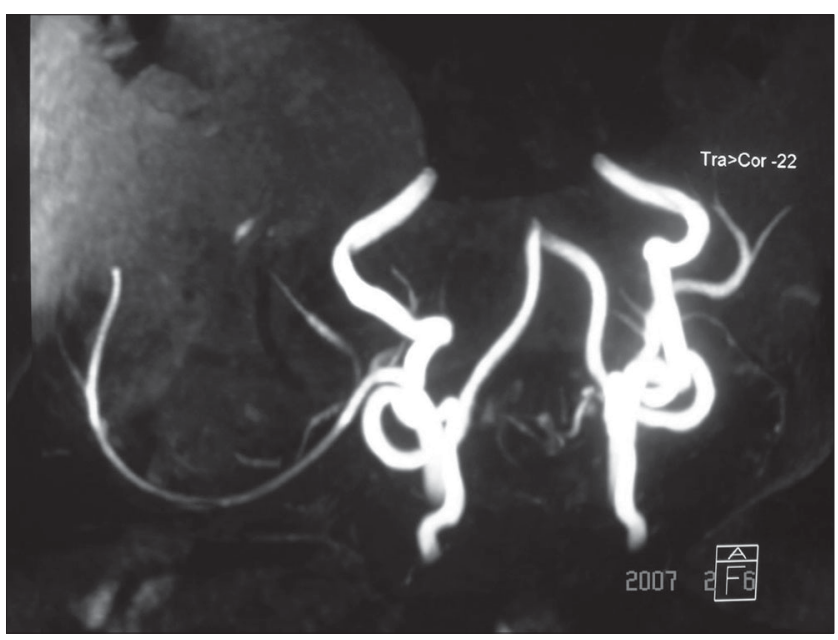

Figure 3: Magnetic resonance angiogram showing stretching of the right external carotid artery around the mass with no significant vascularity

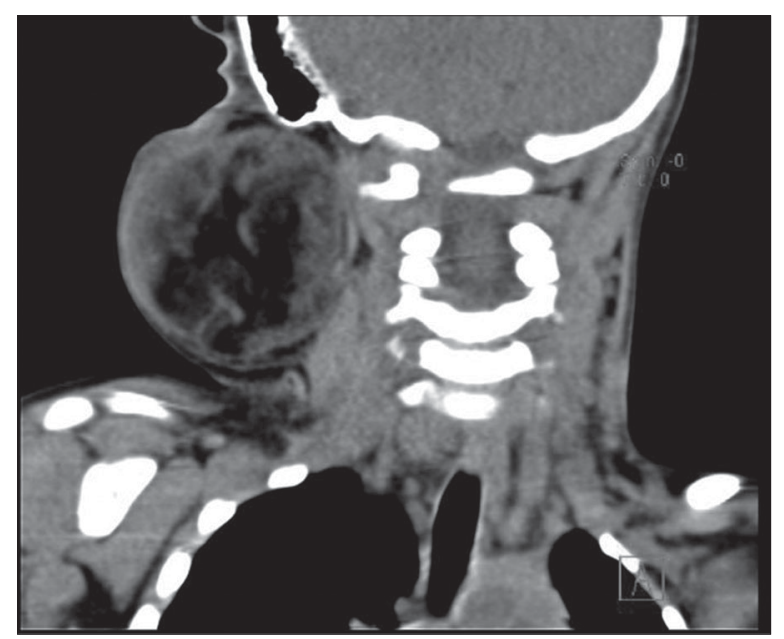

Figure 5: Computerized tomogram scan showed a lobulated hypodense mass in the right infratemporal region ( $\mathrm{HU}$ values are $-50-$ to -75 , indicative of fat) with solid component

out. Mucoperiosteal incision was carried out through the lower buccal sulcus up to the first deciduous molar. The mucoperiosteum distal to the mental nerve was not elevated to preserve the blood supply. The incision was then extended up to the maxillary tuberosity along the maxillary sulcus up to the upper second deciduous molar region at the subperiosteal level. From the deciduous
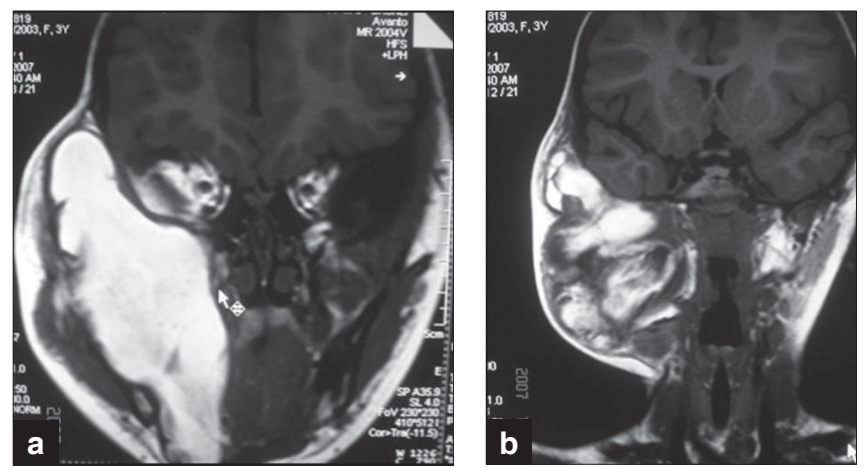

Figure 2: Magnetic resonance imaging scan showing a hyperintense mass on T1-weighted sequence and T2-weighted sequence in the right infratemporal fossa extending along the ramus of the mandible and infiltrating the parotid gland of the lesion

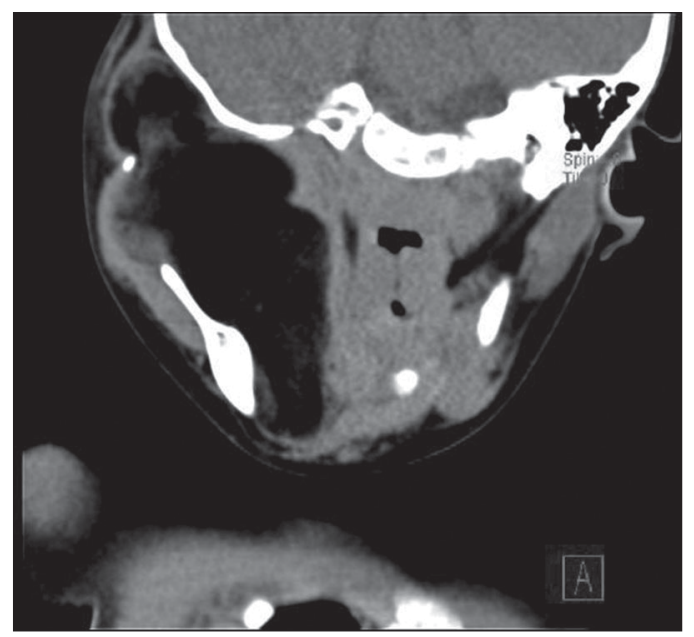

Figure 4: Computerized tomogram scan showed a lobulated hypodense mass in the right infratemporal region (HU values are -50 - to -75 , indicative of fat) with solid component

molar region, the lingual mucoperiosteal flap was elevated along the gingival margins of the teeth up to midline. Paramedian mandibulotomy was followed by lateral swing of the mandible. A large mass was found in the infratemporal fossa extending superiorly to the skull base and inferiorly to the neck. Superiorly, the lesion was dissected off the internal carotid artery. The infratemporal mass was completely removed. Thereafter, attention shifted to removal of the parotid gland, including its deep lobe, involved by the tumour [Figure 7]. Through a pre-auricular incision, the skin flap was elevated, and tragal pointer and bony meatus were identified. The main trunk of the facial nerve was found displaced inferiorly and since the mastoid process was hypoplastic, the nerve was very superficial. Total parotidectomy was done, meticulously preserving the facial nerve and its branches. After surgery, the child recovered well. At 3-years followup there was no recurrence. 


\section{Pathology}

Gross pathological examination revealed a circumscribed lobulated fatty mass measuring $11 \mathrm{~cm} \times 8 \mathrm{~cm}$ [Figure 8]. Histological examination revealed a circumscribed mass formed of mature fat cells and small lipoblasts of varying size and multiple foci of myxoid degeneration. The lobules in the mass were well demarcated due to thick fibrous
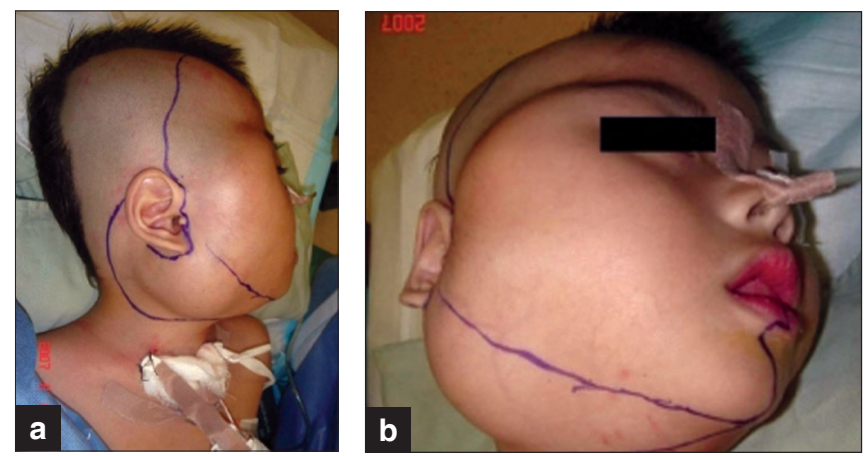

Figure 6: Surgical incision markings

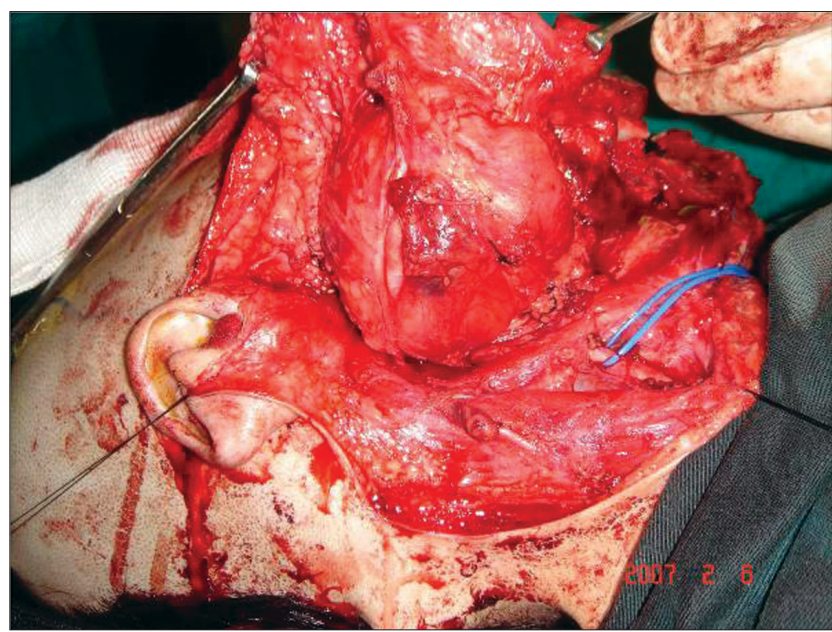

Figure 7: Infratemporal mass before excision

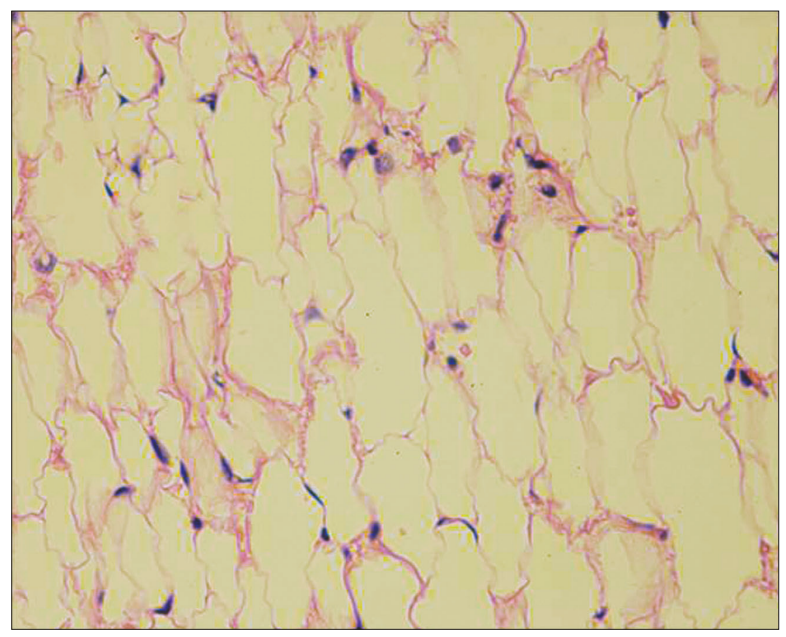

Figure 9: Histopathology revealed mature fat cells and small lipoblasts of varying size and multiple foci of myxoid degeneration septae [Figure 9]. The mass was seen to have completely replaced the parotid gland with lipoblastoma cells.

\section{DISCUSSION}

Lipoblastoma is a rare benign tumour of embryonal fat. Jaffee $^{[1]}$ first introduced the term lipoblastoma in 1926 to describe a tumour of immature fat. Chung and Enzinger ${ }^{[2]}$ described two forms - the term lipoblastoma for the localised circumscribed type and lipoblastomatosis for the diffuse multicentric variant. It occurs more exclusively in infants and children, with $90 \%$ of the cases in children $<3$ years of age. It shows a male preponderance $(3: 1)$. $^{\mid 2]}$ Lipoblastomas commonly arise from the extremities (36$72 \%)$, trunk (20-50\%) and in the head and neck (10-15\%). ${ }^{[2]}$

To date, 34 cases of cervical lipoblastomas have been described in the English literature.

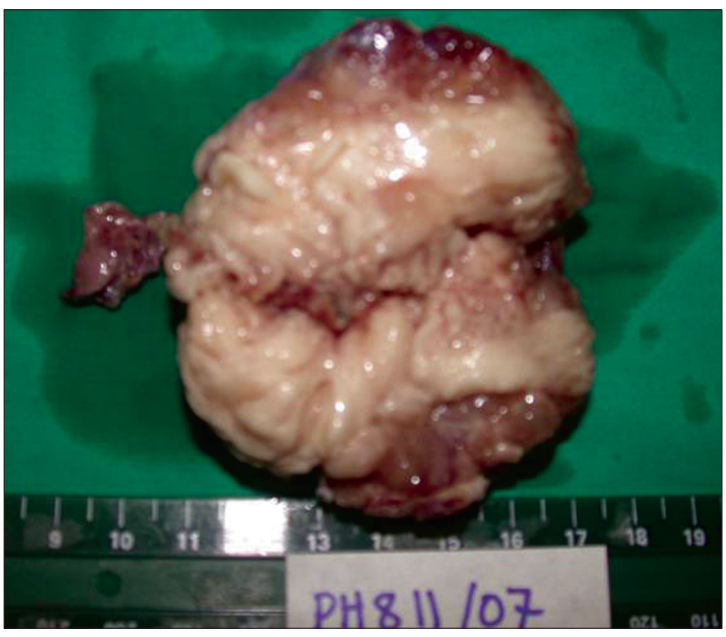

Figure 8: Excised specimen

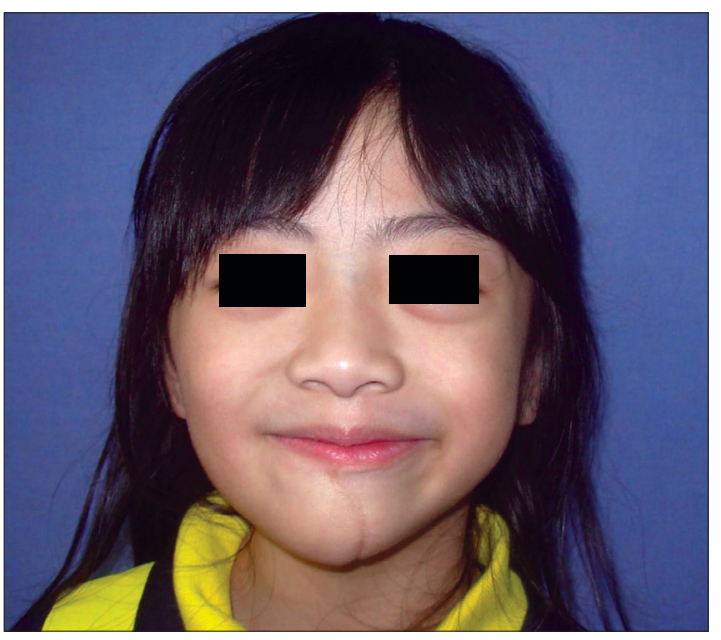

Figure 10: Three-year postoperative picture 
Only two cases of lipoblastoma arising from the salivary gland have been described so far. Calhoun et al. ${ }^{[3]}$ reported a parotid lipoblastoma involving the superficial lobe of a 7-month-old male infant.

Krempl et al., ${ }^{[4]}$ in 1997, described lipoblastoma arising from the deep lobe of the parotid in a 13-month-old male infant.

In the cervical region, the most common presentation is a rapidly growing painless mass. Imaging studies have been used pre-operatively to assess the lesion. CT scan usually shows a lesion in which the Hounsfield density is consistent with fat ${ }^{[5]}(\mathrm{HU}-50$ to -75$)$.

MRI shows a hyperintense image on T1-weighted and T2-weighted sequences, but shows lower signal intensity than mature fat tissue (lipoma) on T1-weighted images. The fibrous septae appear hypointense, making the tumour homogeneous. The high signal intensity on the T2-weighted sequence may be caused by the rich myxoid stroma with numerous lipoblasts. ${ }^{[6]}$ However, none of these findings are diagnostic of lipoblastomas because all these features can be observed in many benign and malignant fatty tumours. MRI is particularly useful for follow-up and evaluation of recurrent lesions.

Lipoma, hibernoma, liposarcoma and embryonal rhabdomyosarcoma are considered in the differential diagnosis of a lipoblastoma. Histologically, lipoblastomas consist of lobulated adipose tissue with fibrous septae with myxoid stroma and immature lipoblasts. Lipomas have lobules with fibrous septae, but lack lipoblasts. Hibernomas also have a lobular pattern, but consist entirely of brown fat cells with eosinophilic granular cytoplasm..$^{[7]}$

Recent cytogenetic analysis revealed specific chromosomal abnormalities in adipose tissue tumours, which may aid in accurate diagnosis. Cytogenetic breakpoint abnormalities ${ }^{[8]}$ occur consistently in the chromosome 8q11-13 in lipoblastomas affecting PLAG1.

Leptin is a hormone that is produced by adipocytes. Leptin and leptin receptors are found in lipoblastomas. Deutscher et al. proposed a hypothesis that the peripheral action of leptin via its receptors could play a role in the development and/or progression of lipoblastoma. ${ }^{[9]}$

Complete surgical excision is the treatment of choice. Incomplete excision would result in recurrence. Hence, careful follow-up is essential for at least a period of 2 years. Three years follow-up in our patient did not reveal any recurrence [Figure 10].

\section{REFERENCES}

1. Jaffe RH. Recurrent lipomatous tumors of the groin: Liposarcoma and lipoma pseudomyxomatodes. AMA Arch Pathol 1926;1: 381-7.

2. Chung EB, Enzinger FM. Benign lipoblastomatosis: An analysis of 35 cases. Cancer 1973;32:482-92.

3. Calhoun $\mathrm{KH}$, Clark WD, Jones JD. Parotid lipoblastoma in an infant. Int J Pediatr Otorhinolaryngol 1987;14:41-4.

4. Krempl GA, McGuff HS, Pulitzer DR, Otto RA. Lipoblastoma in the parotid gland of an infant. Otolaryngol Head Neck Surg 1997;117:S234-7.

5. Stringel G, Shandling B, Mancer K, Ein SH. Lipoblastoma in infants and children. J Pediatr Surg 1982;17:277-80.

6. Reiseter T, Nordshus T, Borthne A, Roald B, Naess P, Schistad O. Lipoblastoma: MRI appearances of a rare paediatric soft tissue tumor. Pediatr Radiol 1999;29:542-5.

7. Enzinger FM, Weiss SW. Benign lipomatous tumors. In: Enzinger FM, Weiss SW, editors. Soft tissue tumors. 3rd ed. Mosby; St. Louis; 1995. p. 401-5.

8. Ohjimi $\mathrm{Y}$, Iwasaki H, Kaneko $\mathrm{Y}$, Ishiguro M, Ohgami A, Kikuchi M. A case of lipoblastoma with $t(3 ; 8)(q 12 ; q 11.2)$. Cancer Genet Cytogenet 1992;62:103-5.

9. Deutscher J, Meyer K, Blütters-Sawatzki R, Franke FE, Kiess W. Leptin and leptin receptor expression in a lipoblastoma in an 8 year old girl. Horm Res 1999;51:253-5.

Source of Support: Nil, Conflict of Interest: None declared. 